\title{
Immature Gastric Teratoma in an Infant: A Case Report
}

\author{
Seong Eon Yoon, $\mathrm{MD}^{1}$ \\ Hyun Woo Goo, MD' \\ Sun-young Jun, MD² \\ In Cheol Lee, MD² \\ Chong Hyun Yoon, MD'
}

Gastric teratomas are extremely rare neoplasms and almost exclusively benign. They occur predominantly in males and generally present as a palpable abdominal mass. To our knowledge, only one adult case has been described in the Korean literature. We report a case in which an immature gastric teratoma in a 3-month-old boy was revealed by CT and US.
Index terms:

Stomach, neoplasm

Infant, teratoma

Korean J Radiol $2000 ; 1: 226-228$

Received June 15, 2000; accepted

after revision August 8, 2000.

Departments of 'Radiology, and ${ }^{2}$ Pathology, Asan Medical Center, University of Ulsan College of Medicine.

Address reprint requests to: Chong Hyun Yoon, MD, Department of Radiology, Asan Medical Center, University of Ulsan College of Medicine, 388-1 Poongnap-dong, Songpa-gu, Seoul 138736 , Korea.

Telephone: (822) 2224-4400

Fax: (822) 476-4719

e-mail: radiology@hanmir.com

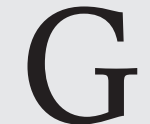

astric teratomas are extremely rare neoplasms. Certain characteristics of these tumors, such as male predominance and a benign nature, are not shared with teratomas found at other sites (1). To date, 100 cases of gastric teratomas, including one in Korea, have been reported worldwide $(2,3)$. The case reported in the Korean literature involved a 26 -year-old man, and pathologically, the tumor showed no immature component (2). We describe a case in which histological examination of a gastric teratoma occurring in a 3-month-old boy disclosed immature neural tissue. To our knowledge, this is the first report of the occurrence of this tumor in an infant to be published in Korea, and worldwide, is the fifth case involving immature tissue to be described.

\section{CASE REPORT}

A 3-month-old boy presented with a palpable abdominal mass. Radiographs of the abdomen disclosed that in the left upper quadrant, a large lesion causing inferomedial displacement of the bowel loops was present. US revealed a large, mixed-echoic, solid and cystic mass in this same area (Fig. 1A), while unenhanced abdominal CT scans depicted a well-defined heterogeneous mass with intratumoral nodular calcifications (Fig. 1B) and a suspicious focal area of fat density. Enhanced abdominal CT scans showed a large, exogastric, solid and cystic mass arising from the greater curvature of the stomach, with continuity between this curvature and the intraluminal extension of the mass. These scans also indicated that the solid portion of the mass was well enhanced (Fig. 1C). The differential diagnosis was germ cell tumor such as teratoma or stromal tumor of the stomach, neuroblastoma, or a mass originating in the tail of the pancreas. A pre-operative diagnosis of teratoma was based on the presence of intratumoral calcification and mixed cystic and solid components of the mass.

The tumor was totally excised, involving partial gastrectomy, and the defect in the stomach was repaired. The gross specimen of the round exogastric mass thus obtained measured $14 \times 9 \times 6.5 \mathrm{~cm}$ and originated from the posteroinferior wall of the stomach along its greater curvature (Fig. 1D). The protruding intraluminal polypoid mass, measuring $7 \times 5 \times 3 \mathrm{~cm}$, formed a continuation of the exogastric mass and was entirely covered by serous membrane. It contained multiple cystic areas filled with serous and mu- 
cinous material, keratin, and hemorrhagic foci. Histologically, the tumor contained not only mature components such as chondroid tissue, squamous epithelium, and keratin, but also multifocal aggregates of immature neural tissues of neuroblasts. The pathologic diagnosis was immature teratoma of the stomach. Three months later, follow-up CT revealed neither evidence of tumor recurrence nor distant metastasis.

\section{DISCUSSION}

Teratomas are neoplasms containing bizzare, highly or- ganized tissues derived from all three germ layers. Their exact cause is not known. In cases of gastric teratoma, the germ cell theory that extragonadal teratomas originate from migrated totipotential germ cells has been generally accepted (2). The most common form of teratoma to occur in infancy and childhood is sacrococcygeal teratoma (60$65 \%$ of cases), followed by gonadal $(10-20 \%)$, mediastinal $(5-10 \%)$, presacral (5\%), and intracranial, retroperitoneal, and cervical $(<5 \%)$ teratomas (3).

Gastric teratoma is an extremely rare neoplasm, accounting for less than $1 \%$ of all teratomas occurring in infancy and childhood. Since the first case of gastric teratoma was
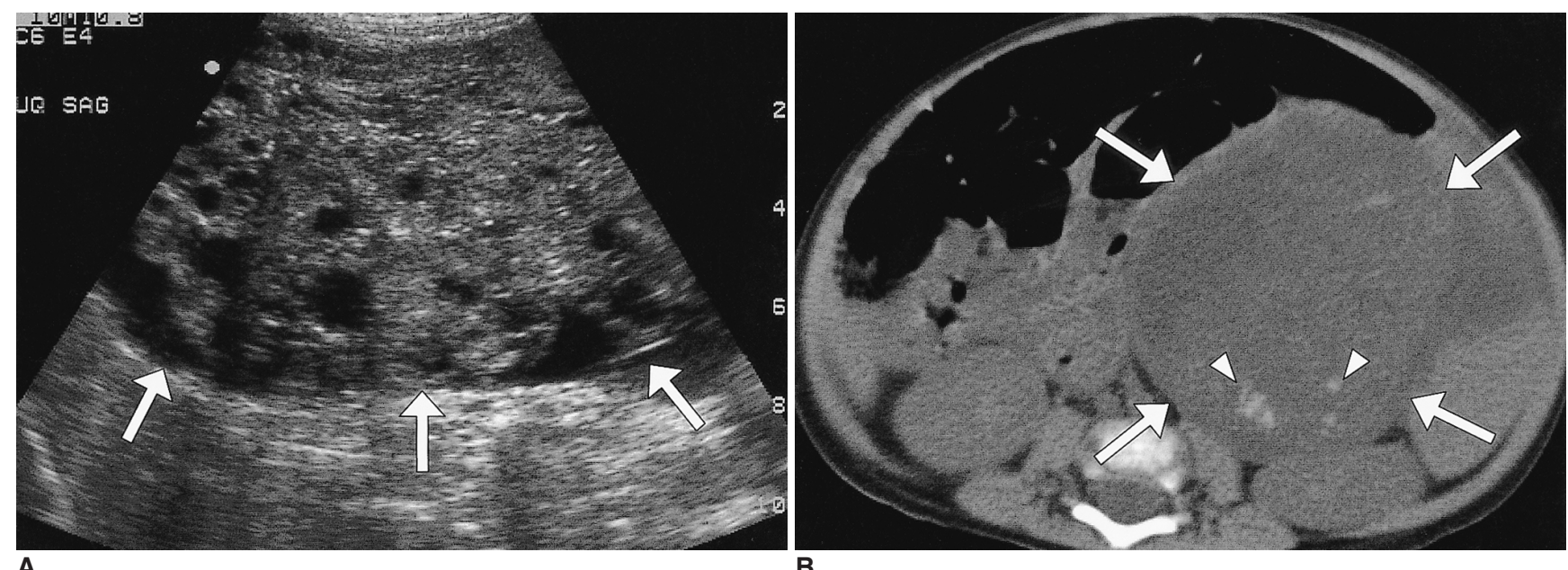

B
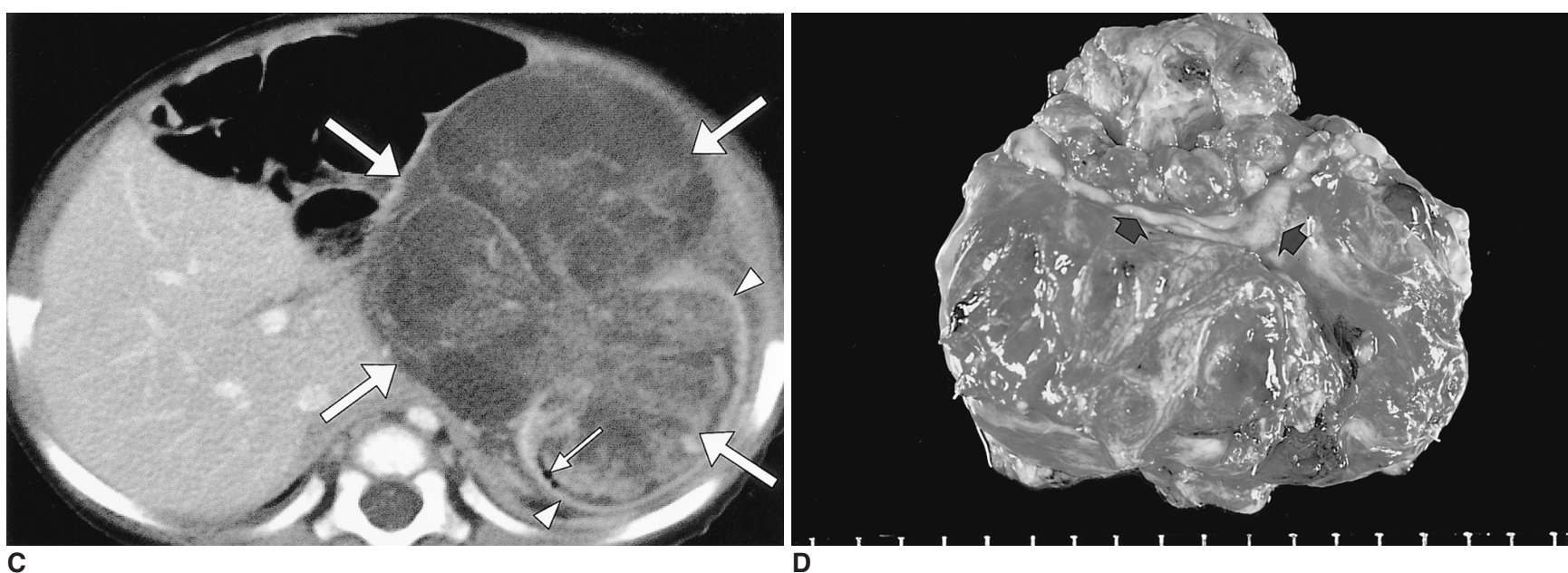

Fig. 1. Radiological and pathological findings of immature gastric teratoma in a 3-month-old boy.

A. Longitudinal US of the abdomen shows a $20 \times 13 \times 9 \mathrm{~cm}$-sized mixed-echoic mass in the left upper abdomen, with multifocal cystic components (arrows).

B. Unenhanced abdominal CT at the level of the kidneys shows a well-defined heterogeneous mass displacing the left kidney posteriorly and the bowel loops medially (arrows). Note the presence of multiple nodular calcifications in the mass (arrowheads).

C. Enhanced abdominal CT at the level of the stomach shows a large exogastric, solid, cystic mass arising from the greater curvature of the stomach, and continuous with a protruding intraluminal polypoid mass (arrows). Note the well-enhanced gastric wall (arrowheads) and the presence of air bubbles in the gastric lumen (small slender arrow).

D. Gross specimen of the round exogastric mass, $14 \times 9 \times 6.5 \mathrm{~cm}$ in size, which originated from the posteroinferior wall of the stomach along its greater curvature (arrows). Cut section of the tumor shows some cystic areas filled with serous and mucous material, and keratin, and the presence of hemorrhagic foci. 
reported in 1922 by Eusterman and Sentry, only 100 cases have been reported in the literature $(1-3)$. In the Korean literature, one case occurring in an adult has been reported, but ours is the first published case to involve an infant. Although gastric teratomas can occur at any age, approximately $94 \%$ of reported case have involved infants or neonates $(2,5)$. There is, in addition, a striking male predominance of gastric teratoma, with only seven cases $(7 \%)$ occurring in females (2).

Almost all reported gastric teratomas have been benign, with no malignant elements in any tumor of any size, or even among those found in adults $(1-5)$. The exception is one case involving malignant transformation, reported by Matsukama et al. (6). To our knowledge, only four cases in which there were immature elements have been described in the English literature (1). Where histologic examination indicates that a tumor is immature, malignancy may or may not ensue, though neither metastasis nor recurrence has been reported. Gastric teratomas are considered benign, with excellent prognosis after total excision (1).

Gastric teratomas may present as a palpable mass $(75 \%$ of reported cases) and/or abdominal distension (56\%). In infants, respiratory distress is the frequent outcome, whereas in adults, epigastric discomfort and hematemisis or melena are the main symptoms $(2,3)$. The growth of gastric teratomas has been solely exogastric in $65 \%$ of reported cases, solely endogastric in $9 \%$, and endo/exogastric in $26 \%$ (1). In the present case, continuity between the exogastric mass and the endogastric polypoid mass along the greater curvature, as revealed by $\mathrm{CT}$, defined the gastric origin of the lesion.

Radiologic evaluation can differentiate between gastric teratoma and other more common abdominal masses of childhood. Plain films usually reveal a soft tissue mass with associated calcifications, if these are present, but are non- specific. US and CT can demonstrate not only a heterogeneous mass containing varying amounts of cystic and solid components, but also fat and calcification. The latter suggest a diagnosis of gastric teratoma. In our case there was no definite evidence of fat components, but other findings such as calcification, and the mixed cystic and solid component of the mass, suggested teratoma. Differential diagnosis should include other pediatric abdominal masses, namely neuroblastoma, Wilms tumor, pancreatic cyst, hepatoblastoma, rhabdomyosarcoma, liposarcoma, and retroperitoneal teratoma (4).

In conclusion, gastric teratoma is an extremely rare tumor of childhood, and in almost all cases is benign. It most commonly occurs in male infants as a palpable abdominal mass, and must therefore, be distinguished from more common causes of abdominal masses in children. Radiologic evaluation can demonstrate the gastric origin of the mass and specific features of teratomas such as fat and calcification, which help to exclude other palpable masses encountered in childhood.

\section{References}

1. Munoz NA, Takehara H, Komi N, Hizawa K. Immature gastric teratoma in an infant. Acta Paediatr Japon 1992;34:483-488

2. Joo M, Kang YK, Lee HK, et al. Intrapulmonary and gastric teratoma: report of two cases. J Korean Med Sci 1999;14:330-334

3. Shirodkar NP, Chopra PS, Marker M, Murphy KD, Dhamoon A, Kwon OJ. Conjoined gastric and mediastinal benign cystic teratomas: case report of a rare occurrence and a review of the literature. Clin Imaging 1997;21:340-345

4. Dunlap JP, James CA, Maxson RT, Bell JM, Wagner CW. Gastric teratoma with intramural extension. Pediatr Radiol 1995;25:383-384

5. Matsukuma S, Wada R, Daibou M, et al. Adenocarcinoma arising from immature gastric teratoma: report of a case in an adult and a review of the literature. Cancer 1995;75:2663-2668

6. Bourke CJ, Mackay AJ, Payton D. Malignant gastric teratoma: case report. Pediatr Surg Intern 1997;12:192-193 